\title{
A State Estimation Algorithm for Hybrid AC/DC Networks with Multi-Terminal DC Grid
}

\author{
Marco Pau, Abhinav Sadu, Suresh Pillai, Ferdinanda Ponci, Antonello Monti \\ Institute for Automation of Complex Power Systems \\ E.ON Energy Research Center, RWTH Aachen University \\ Aachen, Germany \\ (mpau, asadu, spillai, fponci, amonti)@eonerc.rwth-aachen.de
}

\begin{abstract}
Hybrid AC/DC grids have been recently proposed as an efficient solution to accommodate increasing amounts of renewable energy sources in future smart grids. Similarly to the traditional power systems, state estimation algorithms are necessary in AC/DC grids to acquire awareness about the operating conditions of the system and to enable proper operation of the control tools. This paper presents a state estimator tailored for hybrid AC/DC grids with multi-terminal dc grid configuration. The proposed estimator is based on a twostep algorithm, which uses the boundary results of the $\mathrm{AC}$ and the DC grid to enhance the accuracy of the estimation. Results on a simple test network are presented to show the validity of the proposed estimator and to highlight the inherent benefits.
\end{abstract}

Index Terms--AC/DC grids, HVDC, Multi-Terminal DC grids, State Estimation, Voltage Source Converters.

\section{INTRODUCTION}

The need for electrical grids operated with higher flexibility, reliability and efficiency is driving the evolution towards the smart grids. One of the key aspects is the efficient integration of the renewable energy sources. The use of green energy is essential to reduce the dependence from fossil fuels but it also implies technical challenges for the operation of the grid. Another issue is the increasing level of power consumption. Despite the efforts to enhance the efficiency of the electrical systems, the overall power demand is constantly rising and this trend is expected to continue also during next decades [1]. In this context, it is worth noting that existing grids are already often operated close to their limits and many countries have old infrastructures. For these reasons, the design of future smart grids has to rely upon both innovative solutions and an intelligent refurbishment of the network.

One of the solutions advanced for dealing with these issues is the use of DC networks. In transmission grids, High Voltage Direct Current (HVDC) systems are already used for long distance transmission and to connect large generation plants, like offshore wind farms, to the main grid. Most of the existing HVDC systems are based on point to point connections, but larger DC grids with multi-terminal configuration have been proposed to obtain cost effective solutions [2]. Recently, suggestions for the construction of a pan-European overlaying DC "supergrid" have been also presented [3].

Multi-Terminal DC (MTDC) grids are attractive not only for transmission networks, but also for the modernization of distribution systems. The potential advantages deriving from the use of hybrid AC/DC grids at distribution level include a better utilization of the renewable energy sources, an easier integration of Distributed Energy Resources (DERs), like electric vehicles and storage systems, and the possibility to provide support for the control of the AC grid [4]-[6]. The increasing interest around this topic is further confirmed by ongoing research activities [7] and by the presence of projects aimed at the real implementation of experimental DC distribution grids [8].

Similarly to the traditional AC systems, the management of hybrid AC/DC grids has to rely on the accurate knowledge of the operating conditions. To this purpose, hybrid AC/DC state estimators can be used. This paper proposes a novel approach to perform State Estimation (SE) in AC/DC grids, suitable for multi-terminal schemes and for any configuration (i.e. monopole or bipole) or topology of the DC grid. The presented approach is based on iterative Weighted Least Squares (WLS) algorithms running separately on the AC and DC portions of the hybrid AC/DC grid, which exchange the boundary data, with proper consideration of the converter losses, to refine the final SE results. In the following Section the WLS formulation used to perform SE is briefly recalled. In Section III, the models of the AC grid, of the DC grid and of the converter used into the SE algorithm are presented. The overall structure of the proposed SE algorithm is presented in Section IV, while the simulation results, obtained on a 5-bus AC test network with an embedded 3-terminal MTDC network, are reported and discussed in Section V.

\section{State Estimation}

State Estimation (SE) is a mathematical technique aimed at processing the measurements gathered from the field to filter out the errors and achieve an accurate knowledge of the operating conditions of the grid. SE is used since several

(c) 2016 IEEE. Personal use of this material is permitted. Permission from IEEE must be obtained for all other uses. DOI: 10.1109/ISGTEurope.2016.7856278. Publisher version: http://ieeexplore.ieee.org/document/7856278/ 
decades in transmission systems [9] and now is becoming essential also for the management and control of distribution systems [10]. Several approaches can be adopted to perform $\mathrm{SE}$, but the most common ones are based on the WLS method. The general measurement model used is:

$$
\mathbf{z}=\mathbf{h}(\mathbf{x})+\mathbf{e}
$$

where $\mathbf{z}$ is the vector of the telemetered measurements; $\mathbf{x}$ is the vector of the state variables used to describe the grid; $\mathbf{h}$ is the vector of the measurement functions linking the measurements $\mathbf{z}$ to the state vector $\mathbf{x}$; $\mathbf{e}$ is the vector of the measurement errors, which are usually considered as zero mean and decorrelated errors with covariance matrix $\boldsymbol{\Sigma}_{\mathbf{z}}=$ $\operatorname{diag}\left(\boldsymbol{\sigma}_{z}^{2}\right)$, where $\boldsymbol{\sigma}_{z}$ is the vector of the standard deviations of the different measurements. Starting from this measurement model, the WLS method is designed to achieve the minimization of the following objective function:

$$
\mathrm{J}(\mathbf{x})=[\mathbf{z}-\mathbf{h}(\mathbf{x})]^{\mathrm{T}} \mathbf{W}[\mathbf{z}-\mathbf{h}(\mathbf{x})]
$$

where $\mathbf{W}=\boldsymbol{\Sigma}_{z}^{-1}$ is a weighting matrix introduced to provide higher weight to the measurements with better accuracy and vice versa. The minimization of (2) can be obtained by applying the iterative Gauss-Newton method, which leads, for each iteration of the SE algorithm, to the following equation system to be solved:

$$
\mathbf{G} \cdot \Delta \mathbf{x}=\mathbf{H}^{\mathrm{T}} \mathbf{W}[\mathbf{z}-\mathbf{h}(\mathbf{x})]
$$

where $\mathbf{H}$ is the Jacobian of the measurement functions $\mathbf{h}(\mathbf{x})$, $\mathbf{G}=\mathbf{H}^{\mathbf{T}} \mathbf{W} \mathbf{H}$ is the so-called Gain matrix, and $\Delta \mathbf{x}$ is the updating vector of the state variables $\mathbf{x}$. For each iteration $k$ of the SE algorithm, using the solution of (3), the state vector can be then updated using the following:

$$
\mathbf{x}_{k+1}=\mathbf{x}_{k}+\Delta \mathbf{x}_{k}
$$

The iterative process stops when a given convergence criterion is achieved, which is usually chosen as $\max (|\Delta \mathbf{x}|)<\varepsilon$, where $\varepsilon$ is the chosen threshold.

Despite the research efforts devoted to address different challenges associated to the operation of AC/DC grids, so far only few works deal with hybrid AC/DC SE. In [11], a hybrid estimator is presented where the $\mathrm{AC}$ and $\mathrm{DC}$ grids and the $\mathrm{AC} / \mathrm{DC}$ interconnection are modeled through independent subsystems. In [12], an alternative approach is presented where the whole AC/DC network is handled simultaneously by using a decoupled formulation. In [13], a sequential SE algorithm with coupling Gain submatrices between AC and DC grids is presented, whereas [14] proposes a distributed solution based on a Lagrangian relaxation approach. In all the cases, the losses of the converters binding the AC and DC systems are not explicitly taken into account. In [15], the importance of properly modeling the converter losses is highlighted for power flow studies. This paper proposes a new SE algorithm based on a simple two-step WLS procedure that duly considers the converter losses to enhance the accuracy of the SE results. The main goal is to present the design of a novel hybrid AC/DC SE algorithm able to combine features of accuracy and computational efficiency.

\section{AC/DC GRID MODEL}

The hybrid AC/DC grid is seen here as composed of three main parts: the AC grid, the DC grid, and the converter that interfaces AC and DC grids. Fig. 1 shows, as an example, a 5-bus AC network connected through 3 terminals to a 3-bus DC grid. The different components of the AC/DC grid are highlighted by using different colors. In the following, the main features of these subsystems that are relevant for the proposed SE algorithm will be discussed.

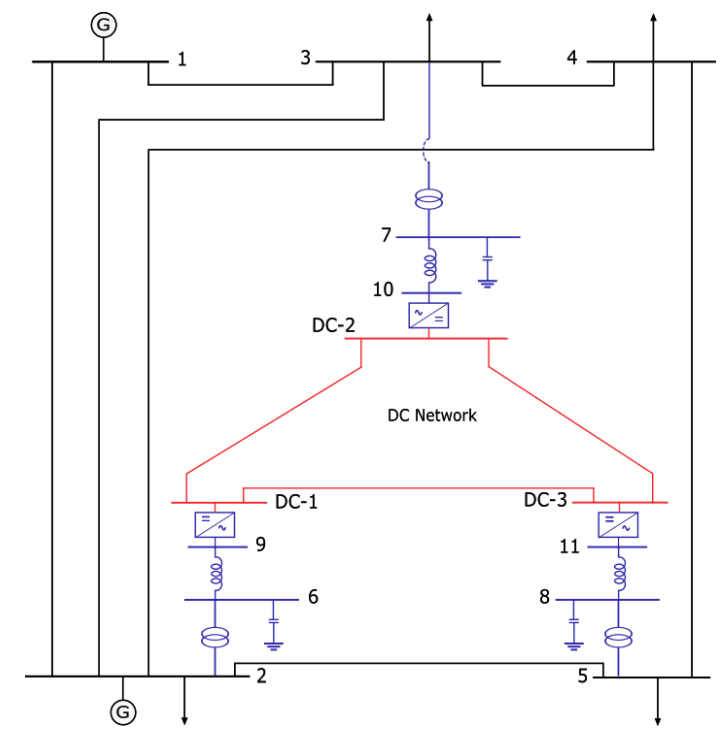

Fig. 1. AC-DC grid model.

\section{A. AC grid}

As shown in Fig. 1, the AC grid is composed of nodes and branches, which can be modeled with the well-known pimodel used in classical power flow studies. From the SE standpoint, the possible measurements $\mathbf{z}_{\mathrm{AC}}$ available in principle in the $\mathrm{AC}$ grid are: voltage magnitude at the buses, current magnitude on the branches, active and reactive power on the branches and active and reactive power injection at the nodes. With the recent deployment of Phasor Measurement Units (PMUs), voltage and current phasor measurements can be also available in addition.

The expression of the functions $\mathbf{h}_{\mathrm{AC}}$ associated to the measurements $\mathbf{z}_{\mathrm{AC}}$ is strictly dependent on the choice of the state vector $\mathbf{x}_{\mathrm{AC}}$. Classical state estimators designed for transmission systems generally use voltage magnitudes and phase-angles as state variables. In this case, the resulting state vector is:

$$
\mathbf{x}_{\mathrm{AC}}=\left[\boldsymbol{\theta}_{\mathrm{AC}}, \mathbf{V}_{\mathrm{AC}}\right]=\left[\theta_{2}, \ldots, \theta_{\mathrm{N}_{\mathrm{AC}}}, \mathrm{V}_{1}, \ldots, \mathrm{V}_{\mathrm{N}_{\mathrm{AC}}}\right]
$$

where $\theta_{i}$ and $V_{i}$ are the voltage phase-angle and magnitude of the $\mathrm{i}$-th node, respectively, and $\mathrm{N}_{\mathrm{AC}}$ is the total number of nodes of the AC grid. However, it is worth noting that in case of distribution systems alternative formulations of the state vector, for example based on the use of branch currents, have been also proposed and they proved to be more efficient than the classical formulation [16]. As a result, a different choice of the state vector can be adopted depending on the 
characteristics of the $\mathrm{AC}$ grid. In this paper, the used formulation is based on polar voltages. However, the proposed SE algorithm is suitable for both transmission and distribution grids, and any choice of the state vector is possible. The expressions of the measurement functions can be found in [9], for the traditional SE based on polar voltages, or in [17], for branch-current state estimators.

\section{B. Voltage Souce Converter}

The power electronic converters used to link AC and DC grids can be divided in two main categories: Current Source Converters (CSC) and Voltage Source Converters (VSC). The CSCs are often used in point to point connections, while VSCs are the most common solution for MTDC grids. As a consequence, in the following, the VSCs will be considered as the interface between AC and DC grid.

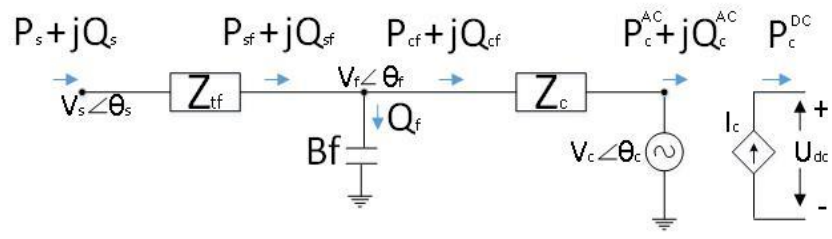

Fig. 2. Equivalent model of a converter station.

The VSC can be modeled as a controllable voltage source connected to the AC grid through a transformer, a capacitive filter and a reactor [15] (see for example the connection in Fig. 1 between nodes 2 and DC-1). Fig. 2 shows a schematic view of the VSC equivalent model, where the impedances of the phase reactor and transformer, and the susceptance of the filter are expressed as $\mathrm{Z}_{c}, \mathrm{Z}_{t f}$ and $\mathrm{B}_{f}$ respectively. As it can be observed, the connection of the converter to the AC grid leads to the presence of two additional nodes on the AC side: the first one at the filter bus, between transformer and reactor, and the second one at the converter bus. In the SE perspective, these two nodes can be considered as additional nodes of the AC grid (as an example, the additional buses for the converter between nodes 2 and DC-1 in Fig. 1 are node 6 and 9). This addition is essential for estimating the electrical quantities on the AC side of the converter, which will be used in the proposed SE algorithm to link the operating conditions of the AC and DC grid. Thus, for each converter, if the classical SE based on polar voltages is chosen, the voltage magnitudes and phase-angles of these nodes have to be added to the state vector $\mathbf{x}_{\mathrm{AC}}$, leading to the following:

$$
\mathbf{x}_{\mathrm{AC}}=\left[\boldsymbol{\theta}_{\mathrm{AC}}, \boldsymbol{\theta}_{\mathrm{f}}, \boldsymbol{\theta}_{\mathrm{c}}, \mathbf{V}_{\mathrm{AC}}, \mathbf{V}_{\mathrm{f}}, \mathbf{V}_{\mathrm{c}}\right]
$$

where $\boldsymbol{\theta}_{\mathrm{f}}$ and $\mathbf{V}_{\mathrm{f}}$ are the vectors of the voltage phase-angles and magnitudes at the filter bus, respectively, and $\boldsymbol{\theta}_{\mathrm{c}}$ and $\mathbf{V}_{\mathrm{c}}$ are the analogous vectors at the converter bus (see Fig. 2). In a similar way, if the branch current SE formulation is used, the two currents flowing through transformer and reactor can be included in the final state vector of the $\mathrm{AC}$ estimator.

As for the possible measurements available on the converter link, since the associated state variables are the same as those used for the AC grid, also the same measurement functions can be used.

Several control strategies can be applied to regulate the electrical quantities of the converter at the AC or DC side. Regardless of the control strategy, the active powers of the converter on the AC and DC side only differ because of the losses. In the proposed estimator the converter active powers and losses are the only quantities used to link AC and DC grid. As a consequence, the converter control does not affect the design of the SE model and the estimator is suited for every kind of control strategy. Indicating with $\mathrm{P}_{c}^{A C}$ and $\mathrm{P}_{c}^{D C}$ the active power of the converter on the $\mathrm{AC}$ and $\mathrm{DC}$ side, respectively, and using as a reference the flow direction shown in Fig.2, the following holds:

$$
\mathrm{P}_{c}^{D C}=\mathrm{P}_{c}^{A C}-\mathrm{P}_{c}^{\text {loss }}
$$

where $\mathrm{P}_{c}^{\text {loss }}$ is the power loss, which depends on the specific features of the converter. Note that eq. (7) is valid regardless of the actual power flow direction and thus it can be used also in case of bi-directional converters. In this study the $\mathrm{P}_{c}^{\text {loss }}$ is modelled as given in eq.(8) [15]:

$$
\mathrm{P}_{c}^{\text {loss }}=a+b \cdot \mathrm{I}_{c}^{A C}+c \cdot \mathrm{I}_{c}^{A C^{2}}
$$

where $a, b$ and $c$ are the loss coefficients characterizing the switching and conduction losses of the converter, and $\mathrm{I}_{c}^{A C}$ is the current magnitude of the converter on the AC side [15]. The converter current can be derived from this equivalent model as per the eq. (9):

$$
\mathrm{I}_{c}^{A C}=\sqrt{\frac{\mathrm{P}_{c}^{A C^{2}}+\mathrm{Q}_{c}^{A C^{2}}}{\mathrm{~V}_{\mathrm{c}}{ }^{2}}}
$$

where $\mathrm{P}_{c}^{A C}$ and $\mathrm{Q}_{c}^{A C}$ are the active and reactive power at the AC converter side, which can be calculated utilizing the state estimates of $\theta_{f}, \theta_{c}, V_{f}, V_{c}$ [15]. In the proposed $S E$ algorithm, it is worth noting that $\mathrm{P}_{C}^{A C}$ and $\mathrm{P}_{c}^{D C}$ represent equivalent power injections for the AC and the DC grid, respectively. The relationships shown in eq. (7), (8) and (9) thus provide a link between the operating conditions of the two grids.

\section{DC grid}

Similarly to the AC grid, also the DC system can be represented through a suitable model of the DC lines. Under steady state conditions, each branch can be simply modeled as a resistive line. The possible measurements $\mathbf{z}_{\mathrm{DC}}$ available on the field can be, in principle: voltage at the nodes, current and power flows on the branches, and current and power injection at the nodes. The corresponding measurement functions $\mathbf{h}_{\mathrm{DC}}$ obviously depend on the set of state variables $\mathbf{x}_{\mathrm{DC}}$ used to describe the system. Similarly to the AC grid scenario, different options could be possible but in the following the vector of the node voltages $\mathbf{V}_{\mathrm{DC}}$ will be considered as state vector:

$$
\mathbf{x}_{\mathrm{DC}}=\mathbf{V}_{\mathrm{DC}}=\left[\mathrm{V}_{1}, \ldots, \mathrm{V}_{\mathrm{N}_{\mathrm{DC}}}\right]
$$

where $\mathrm{V}_{\mathrm{i}}$ is the voltage at the generic node $i$ and $\mathrm{N}_{\mathrm{DC}}$ is the total number of nodes of the DC grid. 
According to this choice of the state vector, it is immediate to see that the voltage measurements can be directly expressed through the same state variables. Instead, current and power measurements on the branch between the generic nodes $i$ (sending node) and $j$ (receiving node) are described by:

$$
\begin{gathered}
\mathbf{h}_{\mathbf{I}_{\mathbf{D C}, \mathrm{ij}}}=\mathrm{g}_{\mathrm{ij}}\left(\mathrm{V}_{\mathrm{i}}-\mathrm{V}_{\mathrm{j}}\right) \\
\mathbf{h}_{\mathbf{P}_{\mathrm{DC}, \mathrm{ij}}}=\mathrm{kg}_{\mathrm{ij}} \mathrm{V}_{\mathrm{i}}\left(\mathrm{V}_{\mathrm{i}}-\mathrm{V}_{\mathrm{j}}\right)
\end{gathered}
$$

where $\mathrm{g}_{\mathrm{ij}}$ is the conductance of the considered line and $\mathrm{k}$ is a coefficient equal to 1 in case of asymmetric monopolar grid or 2 in case of symmetric monopolar and bipolar grid. As for the current and power injection on the generic node $i$, they can be expressed as the sum of the currents or powers, respectively, converging to that node. Thus, this results in the following measurement functions:

$$
\begin{gathered}
\mathbf{h}_{\mathbf{I}_{D C}, \mathrm{i}}=\sum_{\substack{j=1 \\
j \neq i}}^{\mathrm{N}_{D C}} g_{i j}\left(V_{i}-V_{j}\right) \\
\mathbf{h}_{\mathbf{P}_{D C}, \mathrm{i}}=\sum_{\substack{j=1 \\
j \neq i}}^{N_{D C}} \mathrm{~kg}_{\mathrm{ij}} \mathrm{V}_{\mathrm{i}}\left(\mathrm{V}_{\mathrm{i}}-\mathrm{V}_{\mathrm{j}}\right)
\end{gathered}
$$

\section{AC/DC State Estimation AlgORITHM}

The proposed SE algorithm is based on the WLS method and uses the previously presented AC/DC grid model. Fig. 3 shows the flow diagram of the conceived two-step procedure. In the first step, the AC and DC grids are locally estimated, in parallel, using the available AC and DC measurements (together with their uncertainties), respectively. Once the estimated states are obtained, the boundary powers at the converter can be obtained. On the AC side, the boundary power injection $\mathrm{P}_{C}^{A C}$ is first computed (with reference to Fig. 1, the considered boundary nodes for the AC grid are buses 9, 10 and 11). Then, the current magnitude at the converter and the resulting losses are calculated through (9) and (8), respectively. Finally, eq. (7) allows the calculation of the corresponding power injection $\mathrm{P}_{c}^{D C}$ on the $\mathrm{DC}$ grid. The same electrical quantity $\left(\mathrm{P}_{c}^{D C}\right)$ can be computed on the DC grid starting from the DC state estimates. This quantity is exchanged (for each converter) between the AC and DC estimators and is used to obtain an additional power injection measurement at the second step of the AC/DC SE algorithm.

In the second step, a new WLS SE run is performed locally on the AC and DC grids using the power injections provided by the other grid in addition to the locally available measurements. As for the DC grid, the estimation of $\mathrm{P}_{c}^{D C}$ provided by the AC network can be directly used as equivalent power injection. In case of the AC grid, instead, the power losses calculated at the first step are taken into account to obtain the equivalent power injection $\mathrm{P}_{c}^{A C}$ starting from the $\mathrm{P}_{c}^{D C}$ estimation provided by the DC grid (eq. (7)).

It is worth noting that, to properly apply the WLS method, the information on the uncertainty of the additional power injections has to be known (for obtaining the corresponding weights for the second step SEs). To this purpose, the inverse of the Gain matrix is used to obtain the covariance matrix of the estimated states. Then, the following law of propagation of the uncertainty is applied to calculate the uncertainty of the other electrical quantities that are calculated subsequently:

$$
\boldsymbol{\Sigma}_{\mathbf{f}}=\left[\frac{\partial \mathbf{f}}{\partial \mathbf{y}}\right]\left[\boldsymbol{\Sigma}_{\mathbf{y}}\right]\left[\frac{\partial \mathbf{f}}{\partial \mathbf{y}}\right]^{\mathbf{T}}
$$

where $\boldsymbol{\Sigma}_{\mathbf{f}}$ is the covariance matrix of the computed quantities, and $\mathbf{f}(\mathbf{y})$ is the generic function linking these calculated quantities to the starting variables $\mathbf{y}$ (having covariance matrix $\boldsymbol{\Sigma}_{\mathbf{y}}$ ). In the proposed algorithm, (15) is thus used to calculate the uncertainty of the converter powers (starting from the voltage estimations and their covariance matrix) and, similarly, to obtain the uncertainties of converter current magnitude, power losses and, finally, the power injections used in the second step.

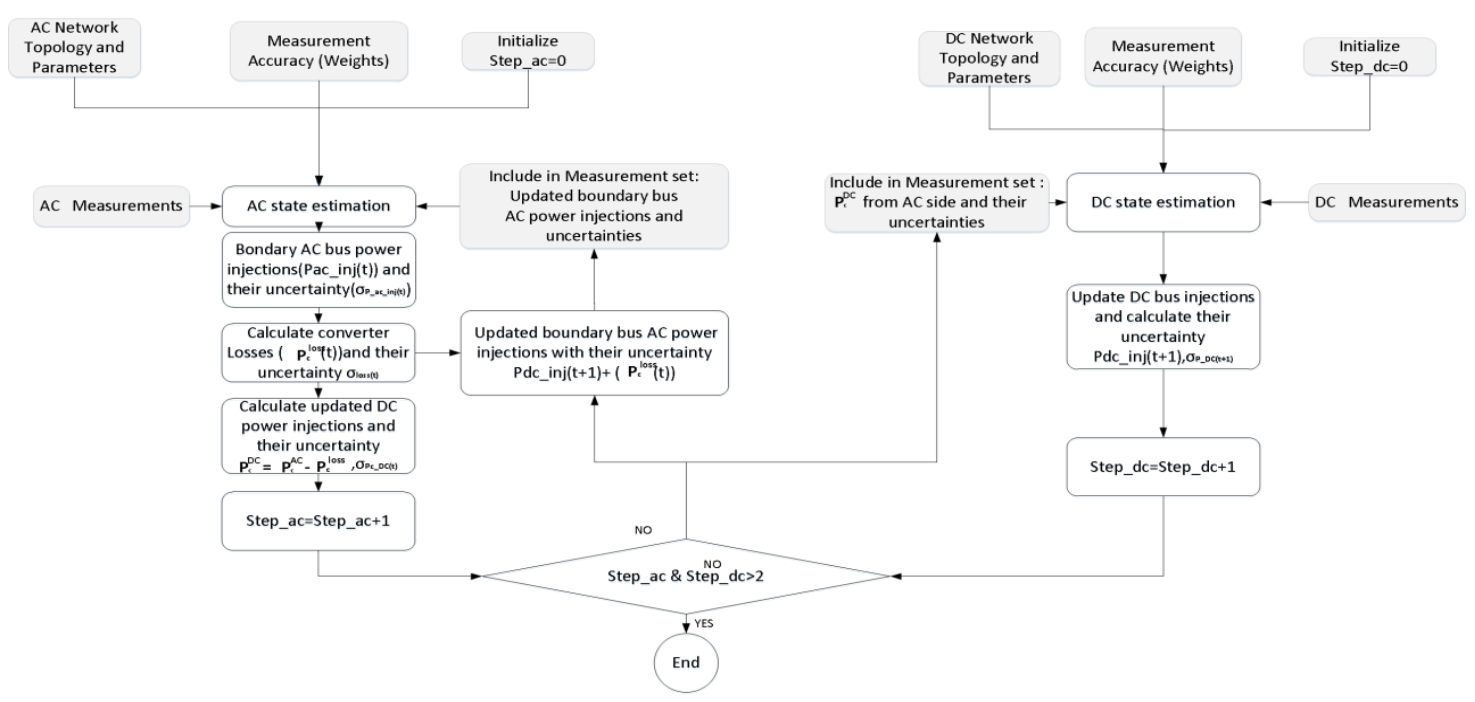

Fig.3. Flow chart of the proposed AC/DC State Estimator 


\section{Simulation Results}

The proposed SE algorithm was implemented in Matlab environment and tested for the hybrid AC/DC grid depicted in Fig.1. Data of the grid can be found in [18]. Simulations were performed using the power flow results obtained through the software in [18] as reference true values and then randomly perturbing them to produce the measurement values in accordance with the assumed measurement uncertainty. Several measurement configurations were tested by means of Monte Carlo simulations to assess the performance of the proposed estimator but, for the sake of brevity, in the following, the presented results focus only on the following measurement configuration (see Fig. 1):

- AC grid: voltage magnitude measurements at nodes 4 and 5; active and reactive power flow measurement for each branch; zero injection at nodes 6, 7 and 8 .

- DC grid: voltage measurement at bus 1; power measurement in the branch between buses 1 and 3; current measurement in the line linking buses 2 and 3 .

- Measurement uncertainty: all the measurements were assumed to have uncertainty equal to $2 \%$ of the reading with Gaussian distributed error and standard deviation equal to one third of that uncertainty.

Monte Carlo tests with 25000 iterations were run in order to compare the statistical results of the two-step AC/DC SE algorithm to those achieved through the local AC and DC estimations. Firstly, the estimation results obtained for the active power flowing through the converters were monitored to evaluate the impact of the second step of the AC/DC estimator. Fig. 4 shows the results in terms of Root Mean Square Errors (in percentage with respect to the considered quantity): it is possible to observe that a significant enhancement is achieved. In particular, it is possible to see that on the DC side, where the measurement redundancy is minimal (according to the assumed measurement configuration), the RMSE associated to the first-step estimation is larger than the one on the AC side and it can be significantly reduced by integrating the more accurate information coming from the AC grid. The benefits of the proposed two-step technique are also confirmed looking at the mismatch between the active power downstream the converters (on the DC side) estimated by the AC and the DC grid (using the same convention for the power flow directions

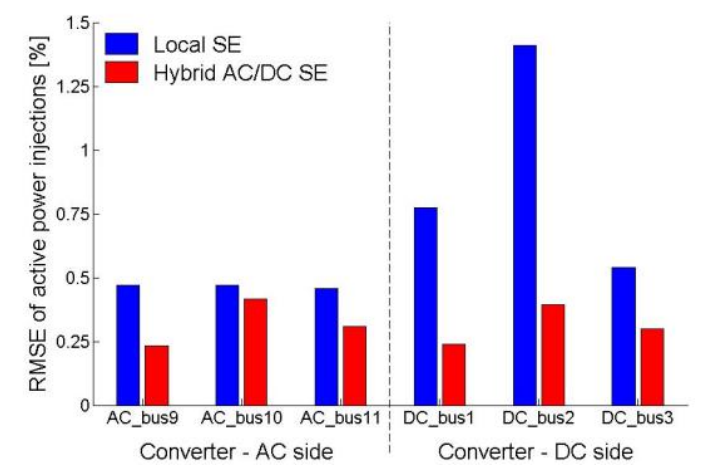

Fig.4. RMSEs for the converter active power on the AC and DC side. as in Fig. 2, the converter losses must be removed from the equivalent power injection estimated on the $\mathrm{AC}$ side). In fact, when running only the local estimates, mismatches in the order of $4-5 \%$ can be found, while when using the hybrid estimator the mismatches reduce to values lower than $0.01 \%$.

The improvements obtained on the boundary powers also affect the other electrical quantities of the two grids. Fig. 5 shows for example the RMSE results obtained for the voltage estimations in the DC grid (in percentage with respect to the reference voltage). It is possible to observe how the addition into the DC estimator of the equivalent active power injections provided by the AC grid (in the second step of the SE algorithm) allows a significant enhancement of the voltage estimation accuracy. For all the buses, improvements larger than $15 \%$ are obtained. Similarly, Fig. 6 shows the outcomes for the voltage magnitude estimations in case of the AC grid. Even in this case, the proposed SE algorithm allows refining the final estimation accuracy. However, the improvements are in this case lower, mainly due to the stronger dependency of the AC voltage magnitude profile from the reactive power rather than the active one.

As explained in Section IV, the WLS method allows obtaining the covariance matrix of the estimated states and, using the law of propagation of the uncertainty, also the uncertainty of all the other electrical quantities inferred from the states can be calculated. Additional tests were thus performed to verify the capability of the AC/DC estimator to correctly provide the uncertainty for the different electrical quantities of the grids after the second step of the algorithm. Obtained results confirm that an almost perfect match exists

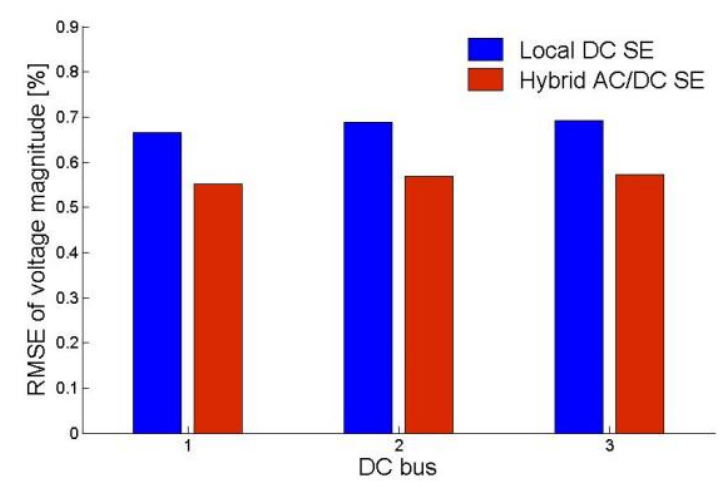

Fig .5. RMSEs for voltage magnitude in the DC grid.

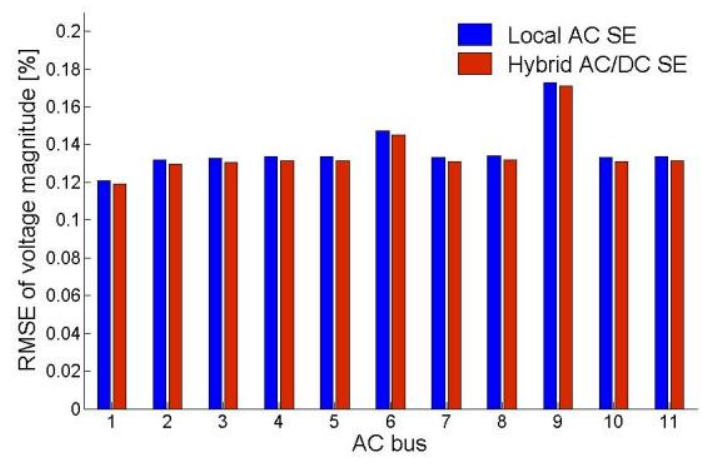

Fig. 6. RMSEs for voltage magnitude in the AC grid. 
between the statistical results given by the Monte Carlo simulation and the theoretical uncertainty computed through the uncertainty propagation law. Table I shows, as an example, the comparison between the statistical and theoretical uncertainty for the voltage estimations on the DC network (since a theoretical uncertainty value is obtained for each Monte Carlo trial, the average, minimum and maximum values obtained over the 25000 trials of the simulation are reported). Analogous results were obtained also for the AC grid and for other different electrical quantities.

TABLE I. Statistical Vs. TheORETiCAL UnCERTAinty

\begin{tabular}{|c|c|c|c|c|}
\hline \multirow{2}{*}{$\begin{array}{c}\text { DC Voltage } \\
\text { Estimation }\end{array}$} & $\begin{array}{c}\text { Monte Carlo } \\
\text { Uncertainty } \\
{[\%]}\end{array}$ & \multicolumn{3}{|c|}{ Theoretical Uncertainty [\%] } \\
\cline { 3 - 5 } & Average & Max & Min \\
\hline DC_bus1 & 1.68 & 1.68 & 1.69 & 1.66 \\
\hline DC_bus2 & 1.71 & 1.70 & 1.72 & 1.69 \\
\hline DC_bus3 & 1.71 & 1.71 & 1.72 & 1.69 \\
\hline
\end{tabular}

As further confirmation of the properties of the proposed estimator, Fig. 7 shows the results of the DC voltage estimation for the first 50 iterations of the Monte Carlo simulation. It is possible to see that the reference true value always lies within the limits of theoretical uncertainty provided by the estimator (a coverage factor equal to 3 is considered for the definition of the uncertainty boundaries).

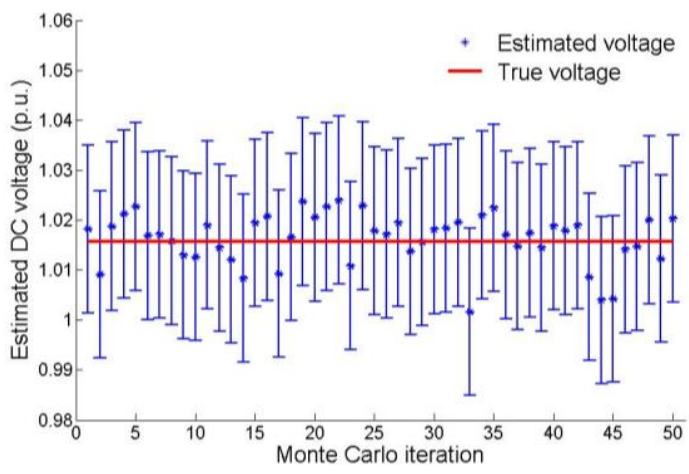

Fig. 7. DC voltage estimation vs. true value over MC iterations.

\section{CONCLUSIONS}

In this study, a two-step SE algorithm for hybrid AC-DC grids with multi-terminal DC grid configuration is presented. Tests were performed to assess the benefits deriving from the exchange of the converter power between AC and DC grids. Results prove that important improvements can be obtained, in particular in grids with low measurement redundancy. The designed AC/DC estimator also performs the calculation of the uncertainty of the estimated quantities, allowing achieving as final output the different electrical quantities of the grid together with their uncertainty. As an extension to this work the authors intend to study the impact of the measurements to find the critical measurements and the configuration of the measurement system for complete observability of the hybrid AC-DC grids. Furthermore the authors intend to improve the presented algorithm to better exploit information about the converter operation modes.

\section{ACKNOWLEDGEMENTS}

This work has been funded by the Bundesministerium für Forschung und Bildung, Forschungscampus Elektrische Netze der Zukunft (FKZ: 03SF0490).

\section{REFERENCES}

[1] EU energy, transport, and greenhouse gas emissions trends to 2050 [Online]. Available: https://ec.europa.eu/energy/en/statistics/energytrends-2050.

[2] W. Lu and B.-T. Ooi, "Optimal acquisition and aggregation of offshore wind power by multiterminal voltage-source HVDC," IEEE Trans. Power Delivery, vol. 18, no. 1, pp. 201-206, Jan 2003.

[3] H. Ergun, J. Beerten and D. Van Hertem, "Building a new overlay grid for Europe," Power and Energy Society General Meeting, 2012 IEEE, San Diego, CA, 2012, pp. 1-8.

[4] D. J. Hammerstrom, "AC Versus DC Distribution Systems - Did We Get it Right?," Power Engineering Society General Meeting, 2007. IEEE, Tampa, FL, 2007, pp. 1-5.

[5] W. Zhang, H. Liang, Z. Bin, W. Li and R. Guo, "Review of DC technology in future smart distribution grid," Innovative Smart Grid Technologies - Asia (ISGT Asia), 2012 IEEE, Tianjin, 2012, pp. 1-4.

[6] A. Mohamed, V. Salehi and O. Mohammed, "Reactive power compensation in hybrid AC/DC Networks for Smart Grid applications," Innovative Smart Grid Technologies (ISGT Europe), 2012 3rd I IEEE PES International Conference and Exhibition on, Berlin, 2012, pp. 1-6.

[7] A. Korompili, A. Sadu, F. Ponci and A. Monti, "Flexible Electric Networks of the Future: Project on Control and Automation in MVDC grids," International ETG Congress 2015; Die Energiewende Blueprints for the new energy age; Proceedings of, Bonn, Germany, 2015, pp. 1-8.

[8] F. Mura and R. W. De Doncker, "Design aspects of a medium-voltage direct current (MVDC) grid for a university campus," Power Electronics and ECCE Asia (ICPE \& ECCE), 2011 IEEE 8th International Conference on, Jeju, 2011, pp. 2359-2366.

[9] A. Abur and A. Gomez-Exposito, Power System State Estimatio. Theory and Implementation. Marcel Dekker, New York, 2004.

[10] D. Della Giustina, M. Pau, P. A. Pegoraro, F. Ponci and S. Sulis, "Electrical distribution system state estimation: measurement issues and challenges," in IEEE Instrumentation \& Measurement Magazine, vol. 17, no. 6, pp. 36-42, December 2014.

[11] A. m. L. Da Silva, G. Perrotta, R. B. Prada and D. M. Falcao, "State Estimation for Integrated Multi-Terminal DC/AC Systems," in IEEE Transactions on Power Apparatus and Systems, vol. PAS-104, no. 9, pp. 2349-2355, Sept. 1985.

[12] A. K. Sinha, L. Roy and H. N. P. Srivastava, "A decoupled second order state estimator for AC-DC power systems," in IEEE Transactions on Power Systems, vol. 9, no. 3, pp. 1485-1493, Aug 1994.

[13] Q. Ding, T. S. Chung and B. Zhang, "An improved sequential method for AC/MTDC power system state estimation," in IEEE Transactions on Power Systems, vol. 16, no. 3, pp. 506-512, Aug 2001.

[14] V. Donde, X. Feng, I. Segerqvist and M. Callavik, "Distributed State Estimation of Hybrid AC/HVDC Grids by Network Decomposition," in IEEE Transactions on Smart Grid, vol. 7, no. 2, pp. 974-981, March 2016.

[15] J. Beerten, S. Cole and R. Belmans, "Generalized Steady-State VSC MTDC Model for Sequential AC/DC Power Flow Algorithms," in IEEE Transactions on Power Systems, vol. 27, no. 2, pp. 821-829, May 2012.

[16] M. Pau, P. A. Pegoraro and S. Sulis, "Performance of three-phase WLS Distribution System State Estimation approaches," Applied Measurements for Power Systems (AMPS), 2015 IEEE International Workshop on, Aachen, 2015, pp. 138-143.

[17] M. Pau, P. A. Pegoraro and S. Sulis, "Efficient Branch-Current-Based Distribution System State Estimation Including Synchronized Measurements," in IEEE Transactions on Instrumentation and Measurement, vol. 62, no. 9, pp. 2419-2429, Sept. 2013.

[18] MatACDC [Online]. Available: www.esat.kuleuven.be/electa/teaching/matacdc/ 